\title{
Gleichgewichte im System: Berylliumoxyd, Oxalsäure und Wasser.
}

\author{
Von \\ Charies L. Parsons und Wm. O. Robinson. ${ }^{1}$ \\ Mit 2 Figuren in Text.
}

\section{Die Berylliumoxalate.}

Bei den Versuchen zur Darstellung von normalem Beryllium-

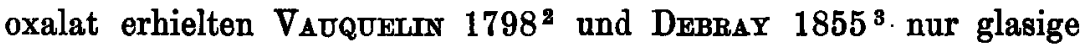
Massen, was darauf zurückzuführen ist, dafs bestimmte kristallisierte Berylliumverbindungen nur herstellbar sind, wenn genau äquivalente Mengen von Kation und Anion vorhanden sind oder wenn letzteres überwiegt. ATtERBERG ${ }^{4}$ exhielt 1873 gleichfalls nur nicht kristallisierte basische Verbindungen, denen er aber bestimmte Formeln $\mathrm{BeC}_{2} \mathrm{O}_{4} \cdot \mathrm{Be}(\mathrm{OH})_{2} \cdot \mathrm{H}_{2} \mathrm{O}$ und $\mathrm{BeC}_{2} \mathrm{O}_{4} \cdot 6 \mathrm{Be}(\mathrm{OH})_{2} \cdot 6 \mathrm{H}_{2} \mathrm{O}$ zuschrieb. Im Jahre 1897 stellten RoseneEIM und WoGE ${ }^{5}$ zuerst das normale Oxalat $\mathrm{BeC}_{2} \mathrm{O}_{4} \cdot 3 \mathrm{H}_{2} \mathrm{O}$ dar und beschrieben ein saures $\mathrm{Salz} 2 \mathrm{BeC}_{2} \mathrm{O}_{4}$. $\mathrm{H}_{2} \mathrm{C}_{2} \mathrm{O}_{4} \cdot 5 \mathrm{H}_{2} \mathrm{O}$, welches, falls es existierte, das erste mit Sicherheit bekannte saure Salz des Berylliums wäre. WYrodBoFF ${ }^{6}$ bestätigte die Resultate von RosENHEIM und WoGE in bezug auf das normale Oxalat, dem ex jedoch eine Formel mit dreiwertigem Beryllium zuerteilte. Auch Doppeloxalate des Berylliumoxalats mit Alkalimetallen sind mehrfach beschrieben worden. Wir haben nun versucht, alle

1 Ins Deutsche übertragen von J. Koppex.

2 Allg. J. d. Chem. 1, 590.

${ }^{3}$ Am. chim. phys. [3] 44, 37.

- Kgl. Svenslia Handl. 12, 51.

S Z. anorg. Chem. 15, 283.

6 Bull. soc. franc. min. 25, 71. 
die oben erwähnten Substanzen und eventuell vorhandene andere Verbindungen der genannten drei Komponenten herzustellen und zu untersuchen.

\section{Reinigung des Materials.}

In einer früheren Mitteilung hatte PAnsons ${ }^{1}$ gezeigt, das dals von Urbatw und Lacombe ${ }^{2}$ hergestellte basische Acetat des Berylliums sehr charakteristische Eigenschaften besälse, die es zur Trennung dieses Elementes von Eisen und Aluminium geeignet erscheinen lassen. Es ist leicht sublimierbar und mehr oder weniger in fast allen organischen Lösungmitteln löslich. Eisen und Aluminium bilden keine ähnlichen Verbindungen und HABER und VAN OORDT ${ }^{3}$ haben bereits vorgeschlagen, ihre normalen Acetate von dem basischen Acetat des Berylliums auf Grund der Löslichkeit des letzteren in Chloroform zu trennen. Parsons 4 hat nun gezeigt, dals heifser Eisessig selbst ein ausgezeichnetes Lösungsmittel für basisches $\mathrm{Be}$ rylliumacetat ist, welches beim Abkühlen der Lösung in schönen Oktaedern auskristallisiert. Dieses Lösungsmittel ist nun besser als alle übrigen zur Trennung des Berylliums von Eisen und Aluminium geeignet, da es die Acetate der letzteren zwar löst, aber beim Erkalten nicht wieder auskristallisieren läfst.

Käufliches Berylliumhydroxyd mit merklichen Mengen von Eisen und Aluminium wurde in Essigsäure gelöst und die Lösung zur Trockne verdampft. Der pulverige Rückstand wurde dann mit Eisessig gekocht und die Lösung durch einen mit Dampf geheizten Trichter filtriert. Beim Abkühlen schied sich basisches Berylliumacetet aus, welches abgesaugt und zweimal mit kaltem Eisessig ausgewaschen wurde. Den Rückstand behandelten wir mit derselben Essigsäure. Dies kann jedoch nicht fortdauernd geschehen, weil das zuerst gebildete Berylliumacetat immer etwas hydrolisiert, so dafs der Rückstand auch nach sorgfältigem Trocknen immer noch Berylliumhydroxyd enthält, welches etwas Wasser an den Eisessig abgibt, wodurch stets ein Verlust an Beryllium in den Mutterlaugen entsteht. Die Kristalle von basischem Berylliumacetat wurden zur Reinigung aus Eisessig umkristallisiert.

\footnotetext{
1 Journ. Am. Chem. Soc. 26, 721.

Compt. rend. 133, 874.

Z. anorg. Chem. 40, 465.

4 Journ. Am. Chem. Soc. 26, 738.
} 
Die bei weitem zweckmäfsigste Verbindung zur Verarbeitung im Laboratorium auf andere Salze ist das basische Berylliumkarbonat. $\mathrm{Da}$ es eine nicht bestimmte Zusammensetzung hat, so mufs sein Berylliumgehalt in jeder Darstellung bestimmt werden, was durch Verglühen im Platintiegel geschieht. Zur Darstellung dieser Substanz wurde das basische Acetat durch heifses Wasser hydrolisiert, durch Zusatz eines geringen Überschusses reiner Essigsäure in Lösung gebracht und die Lösung dann durch Ammoniak gefällt, worauf das Hydroxyd abfiltriert, in Ammoniumkarbonat gelöst und als basisches Karbonat durch Kochen mit Dampf ausgefällt wurde. Der vollkommen weilse und körnige Niederschlag wurde gewaschen und getrocknet. Die Analyse ergab $\mathrm{BeO}=43.03 \%$ und $43.08 \%$

\section{Berylliumoxalat-3-Hydrat, $\mathrm{BeC}_{2} \mathrm{O}_{4} \cdot 3 \mathrm{H}_{2} \mathrm{O}$.}

Basisches Berylliumkarbonat wurde mit einem geringen Überschufs Oxalsäure behandelt, worin es sich leicht unter Erwärmen löst. Beim Verdampfen schieden sich zuerst nadelförmige Kristalle von Oxalsäure aus, nach deren Entfernung beim weiteren Einengen orthorhombische Kristalle von Berylliumoxalat auskristallisierten, die jedoch beträchtliche Mengen Oxalsäure enthielten. Die Kristalle enthielten auch nach 9 maligem Umkristallisieren unter stetiger Entfernung der mitkristallisierenden Oxalsäure noch einen Überschufs der letzteren, wie zahlreiche Analysen zeigten. Sowohl RosEnHEIM und Woge als auch WrrouboFr gaben an, dafs sie zwei Kristallformen des normalen Berylliumoxalats bebachtet hätten; einmal Nadeln und zweitens Platten. Es ist bemerkenswert, dals wir nur die letzteren Kristalle erhielten, und dafs die ersten Anschüsse derselben immer mit nadelförmig kristallisierter Oxalsäure verunreinigt waren. Wir konnten nach den beschriebenen Verfahren nicht zu reinem Oxalat kommen.

Dieses Salz erhielten wir jedoch in schönen orthorhombischen Kristallen durch Zusatz der genau zur Neutralisation der freien Oxalsäure erforderlichen Menge von basischem Berylliumkarbonat zu dem schwach sauren Oxalat. Die Kristalle gaben bei der Analyse Werte, die genau auf die Formel $\mathrm{BeB}_{2} \mathrm{O}_{4} .3 \mathrm{H}_{2} \mathrm{O}$ stimmten.

Es mufs sehr darauf geachtet werden, dafs kein Überschufs des Karbonats verwendet wird, da dieser die Kristallisation vollständig verhindert, so dafs beim Verdunsten nur dicke, gummiartige Massen entstehen. Schon $0.1 \%$ überschussiges Oxyd hebt das 
Kristallisationsvermögen auf und bisweilen genügte schon der sehr geringe Oxalsäureverlust beim Lösen und Eindampfen des normalen Oxalats, die Kristallisation völlig zu verhindern. Zusatz kleiner Kristalle des normalen Oxalats zu der nicht kristallisierten Masse beeinflufsten die Resultate in keiner Weise.

Berylliumoxalat ist bei gewöhnlicher Temperatur stabil. Es löst sich in weniger als seinem eigenen Volumen Wasser bei $100^{\circ}$ und ist nur etwas weniger löslich bei gewöhnlicher Temperatur. Seine Reaktion ist, wie die aller normalen Berylliumsalze, stark sauer; es hat einen ziemlich scharfen sülsen Geschmack und zersetzt sich leicht in der Hitze. Es löst leicht eine grofse Menge Berylliumkarbonat, wobei sich die später beschriebenen flüssigen und festen basischen Lösungen bilden.

Durch die Freundlichkeit von Prof. Samuge L. Penfieud und F. H. Heath, welche für uns die Kristalle freundlichst untersuchen, sind wir in der Lage die folgende kristallographische Charakteristik hinzuzufügen:

$$
\text { Kristalle von } \mathrm{BeC}_{2} \mathrm{O}_{4} \cdot 3 \mathrm{H}_{2} \mathrm{O} \text {. }
$$

Die Kristalle sind orthorhombisch, ihre allgemeine Entwickelung zeigt die beistehende Figur. Folgende Formen wurden beobachtet: $c$ (001), $d$ (101) und $p$ (111).
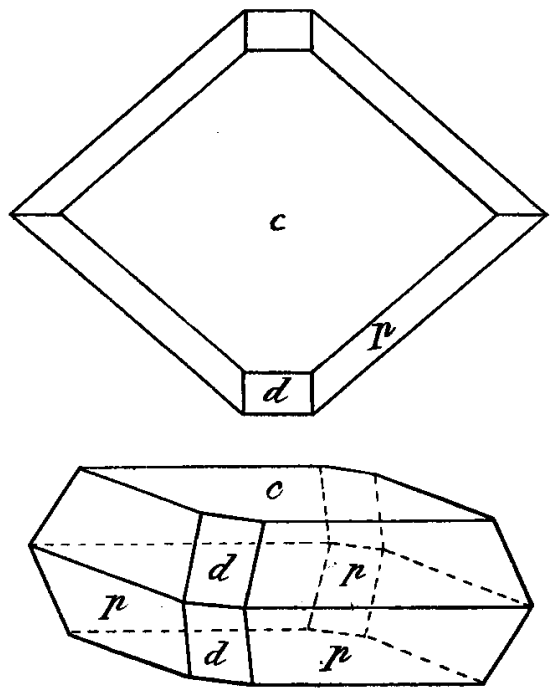

Die Kristalle waren nicht sehr gut für die Messung geeignet; doch konnten die folgenden Beobachtungen gemacht werden: 


$$
\begin{gathered}
-182= \\
p \wedge p^{\prime \prime \prime}, 111 \wedge 1 \overline{1} 1=74^{\circ} 16^{\prime *} \\
p \wedge p^{\prime}, \quad 111 \wedge \overline{1} 11=90^{\circ} 6^{\prime *} \\
c \wedge p, \quad 001 \wedge 111=68^{\circ} 30^{\prime}
\end{gathered}
$$

Berechnet $68^{\circ} 20^{\prime}$.

Die mit einem Stern bezeichneten Zahlen ergeben das Achsenverhältnis $a: b: c 0.853: 1.645$.

Die $d$-Flächen waren so klein und schlecht entwickelt, dafs sie keine genügenden Reflexe gaben. An einen zweiten Kristallanschurs fehlten die $d$-Flächen ganz und die Basis war stärker entwickelt wie gezeichnet ist, so dals die Kristalle als flache Tafeln mit abgeschrägten Ecken erschienen.

Bestimmte Spaltbarkeit war nicht zu erkennen. An einem flachen Kristall zeigte sich unter dem Polariskop, dals die $c$-Achse Bisectrix ist und dafs die Ebene der optischen Achsen mit dem Brachypinacoid zusammenfällt. Die Interferenzfigur war jedoch sehr unbestimmt und der Achsenwinkel so grols, dafs die Hyperbeln sich aufserhalb des Gesichtsfeldes öffneten.

\section{Berylliumoxalat-1-Hydrat, $\mathrm{BeC}_{2} \mathrm{O}_{0} \cdot \mathrm{H}_{2} \mathrm{O}$.}

Dieses Oxalat wird leicht erhalten durch Erhitzen des 3-Hydrats auf $100-105^{\circ}$, bei welcher Temperatur es ebenso wie bei tieferen Temperaturen in trockener Luft beständig ist. Erhitzt man es über diese Temperatur, so beginnt es langsam Wasser zu verlieren, während bei $220^{\circ}$ etwa schneller Wasserverlust eintritt. Oberhalb $220^{\circ}$ beginnt Zersetzung und bei $350^{\circ}$ ist alles in Oxyd umgewandelt. Zwischen der Bildung des wasserfreien Oxalats und seiner Zersetzung liegt kein bestimmtes Intervall; vielmehr beginnt die Zersetzung bereits, bevor alles Wasser ausgetrieben ist, ebenso wie beim Sulfat.

\section{Saures Berylliumoxalat.}

Das von ROSENHEIM und WoGE ${ }^{1}$ beschriebene saure Oxalat konnte nicht erhalten werden, obwohl wir ihren Angaben genau folgten und mehrfach Abänderungen trafen. Ein einziger Versuch genügt zur Charakteristik. Berylliumkarbonat wurde in der doppelt äquivalenten Menge Oxalsäure gelöst und die Lösung über Schwefelsäure fraktioniert kristallisiert. Zuerst schied sich Oxalsäure aus,

1 Z. anorg. Chem. 15, 283. 
sodann Oxalsäure neben normalem Oxalat, welches höchstens 1.18 Mol. Oxalsäure auf $1 \mathrm{Mol}$. BeO enthielt, was durch Einschlufs bedingt war. Da die Kristallarten leicht zu trennen waren, erübrigte sich die Untersuchung der Gleichgewichte, die über die Existenz des sauren Oxalats Auskunft gegeben hätte.

Demnach scheint ein Oxalat $2 \mathrm{BeC}_{2} \mathrm{O}_{4} \cdot 2 \mathrm{H}_{2} \mathrm{C}_{2} \mathrm{O}_{4}$ nicht zu existieren.

\section{Basische Berylliumoxalate.}

Parsons ${ }^{1}$ hat bereits auf die Fähigkeit der Lösungen aller Berylliumsalze hingewiesen, grofse Quantitäten von Berylliumbydroxyd lösen zu können, wobei sich stark basische Lösungen bilden, die allerdings noch saure Reaktion zeigen und welche meistens durch Verdünnen mit Wasser gefällt werden, nachdem eine bestimmte Konzentration an $\mathrm{BeO}$ erreicht ist. Das Oxalat verhält sich ebenso wie das Sulfat in dieser Beziehung und gibt sowohl lösliche als unlösliche basische Substanzen unbestimmter Zusammensetzung.

\section{Lösliche basische Oxalate.}

Wird eine heifse gesättigte Lösung von Oxalsäure mit einem Überschufs von basischem Berylliumkarbonat oder Hydroxyd behandelt, so werden diese gelöst, bis eine Konzentration ron $2.85 \mathrm{BeO}$ auf $1 \mathrm{C}_{2} \mathrm{O}_{3}$ erreicht ist, wobei Kohlendioxyd frei wird und eine dicke syrupöse Lösung entsteht, welche gegen Lakmus noch sauer reagiert. Beim Trocknen wird sie mehr und mehr viskos und schliefslich glasig. Beim Verdünnen mit Wasser entsteht ein weifser voluminöser Niederschlag. Je geringer die Konzentration der benutzten Oxalsäurelösung ist, um so geringer ist auch die relative Menge der gelösten Basis. Alle basischen Lösungen aber geben beim Verdampfen glasige Rückstände, die sich nur dadurch unterscheiden, dafs die basischeren Verbindungen nur in sehr wenig Wasser löslich sind, während die Verbindungen mit weniger als $1.5 \mathrm{BeO}$ auf $1 \mathrm{C}_{2} \mathrm{O}_{3}$ sich in jeder beliebigen Menge Wasser lösen.

Diese Verhältnisse werden durch den folgenden Versuch klar gelegt. $2 \mathrm{ccm}$ einer Lösung mit $2.85 \mathrm{Mol} . \mathrm{BeO}$ auf $1 \mathrm{C}_{2} \mathrm{O}_{3}$ wurden mit je $10 \mathrm{ccm}$ Wasser behandelt, bis die Konzentration von 1 auf 0.01 gesunken war, dann mit je $100 \mathrm{ccm}$ Wasser. Jeder Wasserzusatz

1 Journ. Am. Chem. Soc. 26, 1437. 
bewirkte eine Fällung, bis eine Konzentration von 0.001 erreicht war, worauf weitere Verdünnung ohne Einflufs zu sein schien und das Verhältnis von Basis zu Säure ungefähr 1.5:1 blieb. Beim Verdampfen der Lösungen irgendwelcher Konzentration bildete sich eine leimige Haut, die dann erhärtete und glasartig wurde.

Die folgende Tabelle zeigt die Zusammensetzung dieser löslichen Oxalate. Die Konzentration ist in der ersten Kolumne dargestellt durch das Verhältnis der Kubikzentimeter der gesättigten Lösung zu den Kulikzentimetern Wasser.

Konzentration
$1: 5$
$1: 50$
$1: 100$
$1: 1000$

$$
\begin{gathered}
\text { Verhaltnis } \frac{\mathrm{BeO}}{\mathrm{C}_{2} \mathrm{O}_{3}} \\
2.1: 1 \\
1.9: 1 \\
1.74: 1 \\
1.5: 1
\end{gathered}
$$

Diese Resultate wurden bei gewöhnlicher Temperatur erhalten, und da die Reaktion mit der Verdünnung immer langsamer wird, so ist es wahrscheinlich, dafs nirgends völliges Gleichgewicht erreicht war.

Das Oxalat 2:1 ist dasjenige, dem AtTerberG ${ }^{1}$ die Formel $\mathrm{BeC}_{2} \mathrm{O}_{4} \cdot \mathrm{Be}(\mathrm{OH})_{2} \cdot \mathrm{H}_{2} \mathrm{O}$ zuerteilt; es ist jedoch in keinem Fall eine bestimmte chemische Verbindung.

\section{Unlösliche basische Oxalate.}

RosEnheIM und Woae konnten keine basischen Oxalate erhalten, in denen das Verhältnis $\mathrm{BeO} \quad \mathrm{zu}_{2} \mathrm{C}_{3} \mathrm{O}_{3}$ gröfser als $2.8 \mathrm{zu} 1$ war. Dies kann nur an mangelhaften Versuchsbedingungen gelegen haben, denn diese hochbasischen Niederschläge werden beim Verdünnen mit Wasser aus jeder Oxalatlösung ausgefällt, die mehr als $1.5 \mathrm{BeO}$ auf $1 \mathrm{C}_{2} \mathrm{O}_{3}$ enthält. Die für den Beginn der Fällung erforderliche Verdünnung ändert sich umgekehrt, wie die Basizität der Lösung. Die Wirkung ist in jeder Beziehung ähnlich, wie bei den basischen Sulfaten, nur findet die Fällung nicht, wie dort, scheinbar stufenweise statt.

Die Analysen dieser basischen Fällungen ergaben eine wechselnde Zusammensetzung. Gleich nach der Herstellung zeigten sie ein Verhältnis $10: 1$, welches sich jedoch beim Waschen mit Wasser vergröfserte, obwohl sich die Säure weniger leicht auswaschen liefs, als frther bei den Sulfaten.

1 Kgl. Svenska Akad. Handl. 12, 51. 
Ebenso wie dort, ergab sich bald, dals die Niederschläge nicht vollständig von der Mutterlauge zu trennen waren und deswegen war es durchaus nicht sicher, ob ein einziges basisches Salz oder zwei verschiedene \$alze vorhanden wären, die sich durch Wasser zersetzten. Diese Frage kann jedoch leicht durch Anwendung der Phasenregel gelöst werden, wie MILLER und KENDRICK ${ }^{1}$ gezeigt haben.

Es liegt hier ein System von drei Komponenten vor, und wenn dieses bei bestimmter Temperatur zum Gleichgewicht gekommen ist, so sind aller Wahrscheinlichkeit nach nicht mehr als drei Phasen vorhanden, wenn man die Gasphase nicht berücksichtigt. Wenn also bei einer Reihe von Versuchen die Mutterlauge eine konstante Konzentration hat, während die Zusammensetzung der Niederschläge wechselt, so ist der Bodenkörper ein Gemisch zweier Phasen. Wechselt die Zusammensetzung der Mutterlauge, während die des Bodenkörpers konstant bleibt, so ist. der letztere eine chemische Verbindung. Ändern Lösung und Bodenkörper gleichzeitig ihre Zusammensetzung, so liegt eine feste Lösung, d. h. eine homogene feste Phase variabler Zusammensetzung vor.

Um den fraglichen Punkt aufzuklären, wurde eine Reihe von Versuchen in derselben Weise, wie bereits beim Berylliumsulfat beschrieben, ausgeführt. Die Versuche begannen am 13. April 1905 und das Gleichgewicht war nicht vor dem 10. Juni erreicht. Die Gefälse wurden während dieser ganzen Zeit bei $25^{\circ}$ geschüttelt, während von Zeit zu Zeit Proben für die Analysen entnommen wurden.

(S. Tabelle, S. 186.)

Das angewandte Verhältnis von $\mathrm{BeO} \mathrm{zu} \mathrm{C}_{2} \mathrm{O}_{3}$ ist in der zweiten Kolumne angegeben.

Bei der Prüfung dieser Zahlen ist zu erwähnen, dafs Verhältnisse von $\mathrm{BeO}: \mathrm{C}_{2} \mathrm{O}_{3}$ zwischen $1.5: 1$ und $1: 1$ nicht benutzt werden konnten, weil ist diesem Intervall flüssige und feste Phasen nicht nebeneinander bestehen können.

Aus den angeführten Werten ergibt sich, dafs die Konzentration der Mutterlauge in weiten Grenzen wechselt, weswegen der Niederschlag nicht aus einem Gemisch zweier bestimmter basischer Salze bestehen kann. Es zeigt sich auch, dafs die Konzentration der Mutterlaugen fast immer genau proportional der Anfangskonzentration ist, woraus ebenso wie aus dem Verhältnis von Basis zu

Trans. Roy. Soc. of Canada 7 III, 35. 


\begin{tabular}{|c|c|c|c|c|c|c|c|}
\hline \multirow[b]{2}{*}{ Nr. } & \multicolumn{4}{|c|}{ Angewandt } & \multicolumn{3}{|c|}{ Gefunden } \\
\hline & $\begin{array}{c}\text { Ver- } \\
\text { hältnis } \\
\mathrm{BeO} \\
\mathrm{C}_{2} \mathrm{O}_{3}\end{array}$ & $\begin{array}{l}\mathrm{BeO} \\
\text { in } \mathrm{g}\end{array}$ & $\begin{array}{l}\mathrm{C}_{8} \mathrm{O}_{8} \\
\text { in } \mathrm{g}\end{array}$ & $\begin{array}{l}\mathrm{H}_{2} \mathrm{O} \\
\text { in } \mathrm{g}\end{array}$ & $\begin{array}{l}\mathrm{g} \mathrm{BeO} \\
\text { in } 10 \mathrm{~g} \\
\text { Mutter- } \\
\text { lauge }\end{array}$ & $\begin{array}{l}\mathrm{g} \mathrm{C}_{2} \mathrm{O}_{8} \\
\text { in } 10 \mathrm{~g} \\
\text { Mutter- } \\
\text { lauge }\end{array}$ & $\begin{array}{c}\frac{\mathrm{g} \mathrm{BeO}}{\mathrm{g} \mathrm{C}_{2} \mathrm{O}_{3}} \\
\text { in der } \\
\text { Mutterlauge }\end{array}$ \\
\hline 1 & $2: 1$ & 0.6972 & 1 & 50 & \multirow{3}{*}{\multicolumn{3}{|c|}{$\begin{array}{l}\text { Feste Phase tritt bei dieser Ver- } \\
\text { dünnung und Temperatur }\end{array}$}} \\
\hline 2 & $2: 1$ & 1.0458 & 1.5 & 50 & & & \\
\hline 3 & $2: 1$ & 0.6972 & 1 & 100 & & & \\
\hline 4 & $2: 1$ & $1.0458^{*}$ & 1.5 & 100 & 0.0958 & 0.1501 & $0.64^{*}$ \\
\hline 5 & $21 / 2: 1$ & 0.8715 & $\mathbf{1}$ & 50 & 0.1108 & 0.1847 & 0.60 \\
\hline 6 & $21 / 2: 1$ & 1.3073 & 1.5 & 50 & 0.1765 & - & 一 \\
\hline 7 & $2^{1} / 2: 1$ & 1.7430 & 2 & 50 & 0.2254 & 0.3818 & 0.59 \\
\hline 8 & $21 / 2: 1$ & 0.8715 & 1 & 100 & 0.0567 & 0.0969 & 0.58 \\
\hline 9 & $2^{1 / 2}: 1$ & $1.3073^{*}$ & 1.5 & 100 & 0.0867 & 0.1466 & $0.59 *$ \\
\hline 10 & $21 / 2: 1$ & 2.1788 & 2.5 & 100 & 0.1302 & 0.2361 & 0.55 \\
\hline 11 & $3: 1$ & 1.0458 & 1 & 75 & 0.0750 & 0.1243 & 0.60 \\
\hline 12 & $3: 1$ & 1.5687 & 1.5 & 75 & 0.1228 & 0.2000 & 0.61 \\
\hline 13 & $3: 1$ & 2.0916 & 2 & 75 & 0.1598 & - & - \\
\hline 14 & $3: 1$ & 1.0458 & 1 & 100 & 0.0562 & 0.0969 & 0.58 \\
\hline 15 & $3: 1$ & $1.5687^{*}$ & 1.5 & 100 & 0.0887 & 0.1460 & 0.61 \\
\hline 16 & $3: 1$ & 1.8302 & 1.75 & 100 & 0.0901 & 0.1529 & 0.59 \\
\hline 17 & $4: 1$ & 1.3944 & 1 & 75 & 0.0667 & 0.1257 & 0.53 \\
\hline 18 & $4: 1$ & 2.0916 & 1.5 & 75 & 0.0953 & 0.1793 & 0.53 \\
\hline 19 & $4: 1$ & 1.3944 & 1 & 100 & 0.0480 & 0.0935 & 0.51 \\
\hline 20 & $4: 1$ & $2.0916^{*}$ & 1.5 & 100 & 0.0787 & 0.1465 & 0.54 \\
\hline 21 & $6: 1$ & 2.0916 & 1 & 75 & 0.0505 & 0.1180 & 0.43 \\
\hline 22 & $6: 1$ & 2.0916 & 1 & 100 & 0.0390 & 0.0916 & 0.43 \\
\hline
\end{tabular}

Säure hervorgeht, dafs bei diesen Konzentrationen und bei $25^{\circ}$ die Fällung nur wenig zunimmt, wenn die Verdünnung von 50 auf $100 \mathrm{ccm}$ steigt. Beim Vergleich von Nr. 8, 14, 19 u. 23; von 11, 17 u. 21, sowie von 4, 9, 15, 20 ergibt sich, dals die Zusammensetzung der Mutterlaugen fast allein von der Anfangskonzentration der Säurekomponenten abhängt und nahezu unabhängig von der Basis ist, obwohl eine relativ grofse Zunahme der anfänglichen Basismenge das Verhältnis der Basis in der Mutterlauge herabmindert. Es zeigt sich auch, dafs fast die ganze Säure in Lösung verbleibt, während zunehmend grölsere Beträge Basis in den Niederschlag übergehen, wobei in den meisten Fällen eine Lösung von ungefähr $1.7 \mathrm{BeO}$ zu $1 \mathrm{C}_{2} \mathrm{O}_{3}$ verbleibt. Diese Tatsachen stehen alle in Einklang damit, dafs als feste Phase das Hydroxyd und nicht irgend ein basisches Salz auftritt. 
Schon bald hatte sich gezeigt, dafs ebenso, wie bei den basischen Sulfaten, die Fehler bei der Analyse der Flüssigkeit sich bei der Berechnung so multiplizierten, dafs daraus nichts für die $\mathrm{Zu}$ sammensetzung der festen Phase zu folgern war. Es ist jedoch zu erwähnen, dals solche Berechnungen in jedem Falle Resultate ergaben, die eine viel höhere Basizität anzeigten, als durch direkte Analyse gefunden war, wobei $\mathrm{zu}$ berücksichtigen ist, dals in keinem Falle der Niederschlag vollständig von der Mutterlauge getrennt werden konnte. Die Niederschläge wurden einmal mit Wasser gewaschen, dann zwischen Filtrierpapier geprelst, getrocknet und analysiert. Die Resultate der Analysen an 13 dieser Niederschläge folgen in einer Tabelle.

\begin{tabular}{|c|c|c|c|c|c|c|c|}
\hline $\mathrm{Nr}$. & $\% \mathrm{BeO}$ & $\% \quad \mathrm{C}_{2} \mathrm{O}_{3}$ & $\begin{array}{c}\text { Verhältnis } \\
\frac{\mathrm{BeO}}{\mathrm{C}_{2} \mathrm{O}_{3}}\end{array}$ & Nr. & $\% \mathrm{BeO}$ & $\% \mathrm{C}_{2} \mathrm{O}_{8}$ & $\begin{array}{l}\text { Verhaltnis } \\
\qquad \begin{array}{l}\mathrm{BeO} \\
\mathrm{C}_{2} \mathrm{O}_{3}\end{array}\end{array}$ \\
\hline 8 & 53.2 & 6.2 & 23 & 17 & 50.5 & 6.0 & 26 \\
\hline 11 & 49.7 & 5.5 & 26 & 18 & 48.3 & 6.3 & 22 \\
\hline 12 & 47.8 & 6.5 & 21 & 19 & 49.6 & 6.4 & 22 \\
\hline 13 & 46.3 & 5.1 & 26 & 20 & 51.2 & 5.6 & 26 \\
\hline 14 & 50.6 & 4.4 & 34 & 21 & 51.0 & 5.2 & 28 \\
\hline 15 & 50.1 & 5.3 & 27 & 22 & 52.8 & 4.2 & 35 \\
\hline 16 & 53.2 & 7.0 & 22 & & & & \\
\hline
\end{tabular}

Man erkennt, dafs alle diese Niederschläge beträchtliche Mengen Oxalsäure enthalten, die jedoch nach Analyse der Lösungen wahrscheinlich durch eingeschlossene Mutterlauge bedingt ist. Dieser Schlufs wird auch dadurch gestützt, dafs das vorhandene Wasser in jedem Falle mehr als hinreichend zur Bildung des Hydroxyds ist. Durch das Vorhandensein von Mutterlauge mufs notwendigerweise die feste Phase weniger basisch erscheinen als sie wirklich ist. Nimmt man die Daten wörtlich, so erhält man Formeln, die zwischen $20 \mathrm{Be}(\mathrm{OH})_{2} \cdot \mathrm{BeC}_{2} \mathrm{O}_{4}$ und $34 \mathrm{Be}(\mathrm{OH})_{2} \cdot \mathrm{BeC}_{2} \mathrm{O}_{4}$ wechseln, wobei in jedem Falle ein geringer Überschufs von Wasser vorhanden ist. Die Analysen sind jedoch mit Fehlern behaftet, welche bei der Berechnung solcher stark basischer Produkte einen grofsen Ein. flufs ausüben müssen. Da die Möglichkeit der Gegenwart zweier bestimmter basischer Salze durch Wechsel der Konzentration der Lösungen ausgeschlossen ist, so bleiben noch zwei Fälle übrig. Erstens können die Niederschläge eine feste Phase bestimmter Zusammensetzung sein, die entweder das Hydroxyd selbst oder ein 
basisches Salz ron mindestens $25 \mathrm{BeO}$ auf $1 \mathrm{C}_{2} \mathrm{O}_{3}$ ist; zweitens kann die Zusammensetzung des Niederschlages wechseln und demnach eine feste Lösung geringer Mengen des Oxalats im Hydroxyd vorstellen. Mit Rücksicht auf die bekannte Eigenschaft der Hydroxyde, andere Substanzen mit niederzureifsen, und im Hinblick auf die äufserste Unwahrscheinlichkeit einer Verbindung mit so grofsem Verhältnis von Basis zu Säure, scheint nur die Annahme der letzten Möglichkeit gerechtfertigt.

Die sogenanten "basischen Berylliumverbindungen“.

Wir haben auf den vorhergehenden Seiten den Versuch gemacht, klarzulegen, dafs niemals basische Oxalate oder Sulfate des Berylliums dargestellt sind, und dafs die unbestimmten Gemische, die als solche betrachtet wurden, in der Tat nur feste Lösungen im Hydroxyd seien. Wir zeigten weiter, dafs die beiden Stoffe sich wie Wasser und Alkohol in allen Verhältnissen unter Bildung einer festen homogenen Phase mischen. Aus einer Untersuchung der Einwirkung anderer Säuren auf Berylliumhydroxyd, sowie der Reaktion zwischen Wasser und dem echten basischen Acetat, Formiat, Butyrat, Valerianat usw. sowie durch sorgfältiges Studium aller Originalarbeiten, glauben wir uns zu dem Ausspruch berechtigt, dafs niemals basische Salze des Berylliums dargestellt wurden und niemals dargestellt werden können, wenn Wasser zugegen ist oder wenigstens, wenn es in solcher Menge zugegen ist, dals es einen merkbaren Einflufs auf das System ausüben kann. Es kann mit Sicherheit gesagt werden, dafs keine Bemühungen zur Herstellung bestimmter basischer Salze des Berylliums erfolgreich sein werden, wo man eine Säure mit Berylliumhydroxyd absättigt, wie das in den meisten Fällen geschehen ist, wo man die sogenannten basischen Verbindungen beschrieben hat.

Demnach müssen alle sogenannten basischen Berylliumsalze, die in Gegenwart von Wasser hergestellt worden sein sollen, aus der Literatur gestrichen werden. ${ }^{1}$

Andererseits darf jedoch nicht vergessen werden, dafs die wirklichen basischen, schön kristallisierenden Salze der allgemeinen Formel $\mathrm{Be}_{4} \mathrm{O}(\mathrm{As})_{6}$, worin die Säure entweder Ameisensäure oder

1 Ein Verzeichnis dieser Verbindungen findet sich in der Arbeit von Parsons und Robinson, Journ. Am. Chem. Soc. 1906. 
eine ihrer Homologen ist, zu den interessantesten Verbindungen der anorganischen Chemie gehören, da sie, obwohl basisch, aus wasserfreien Säuren kristallisieren.

\section{Schlufsfolgerungen.}

Die folgenden Schlüsse erscheinen gesichert: Die einzigen bestimmten Hydrate des Berylliumoxalats sind $\mathrm{BeC}_{2} \mathrm{O}_{4} \cdot 3 \mathrm{H}_{2} \mathrm{O}$ und $\mathrm{BeC}_{2} \mathrm{O}_{4} \cdot \mathrm{H}_{2} \mathrm{O}$.

Ein saures Oxalat des Berylliums existiert nicht.

Es ist schwierig, wenn nicht unmöglich, das wasserfreie Berylliumoxalat herzustellen, weil die letzten Spuren Wasser erst dann fortgehen, wenn sich das Oxalat bereits zersetzt.

Die sogenannten basischen Oxalate des Berylliums existieren nicht als besondere chemische Verbindungen, sondern sind in Wirklichkeit feste Lösungen des Oxalats im Hydroxyd. Sie sind viel basischer, nachdem Gleichgewicht mit der Mutterlauge erreicht ist, als nach der ersten Fällung.

Durham, N. H., New Hampshire College, U. S. A.

Bei der Redaktion eingegangen am 26. Februar 1906. 